\title{
Magnetism in $\mathrm{C}_{60}$ Films Induced by Proton Irradiation
}

\author{
S. Mathew, B. Satpati, B. Joseph and B. N. Dev * \\ Institute of Physics, Sachivalaya Marg, Bhubaneswar-751 005, India \\ R. Nirmala and S. K. Malik \\ Tata Institute of Fundamental Research, Mumbai 400 005, India
}

(Dated: November 6, 2018)

\begin{abstract}
It is shown that polycrystalline fullerene thin films on hydrogen passivated $\mathrm{Si}(111)$ substrates irradiated by $2 \mathrm{MeV}$ protons displays ferromagnetic-like behavior at $5 \mathrm{~K}$. At $300 \mathrm{~K}$ both the pristine and the irradiated film show diamagnetic behavior. Magnetization data in the temperature range 2 $300 \mathrm{~K}$, in 1 Tesla applied field, for the irradiated film shows much stronger temperature dependence compared to the pristine film. Possible origin of ferromagnetic-like signals in the irradiated films are discussed.

PACS numbers: 75.70.-i, 81.05.Tp, 61.80.Jh
\end{abstract}

Recent observation of occurrence of ferromagnetism in materials purely of carbon origin ${ }^{1}$ has created enormous interest in these materials. Since 1991, it is known that some organic molecules with unpaired $\pi$ electrons show magnetic order below $20 \mathrm{~K}^{2}$. An antiferromagnetic ordering has been found in a $s-p$ electron system below $50 \mathrm{~K}^{3}$. Makarova et al,$\underline{\underline{4}}$ and Wood et al $\mathrm{l}^{\frac{5}{5}}$ showed the occurrence of ferromagnetic ordering in two dimensionally polymerized highly oriented rhombohydral $\mathrm{C}_{60}(\mathrm{Rh}-$ $\mathrm{C}_{60}$ ) phase. Tetrakis(diemethylamino)ethylene (TDAE) doped fullerides are known to be ferromagnetic below a Curie temperature $\left(\mathrm{T}_{c}\right)$ of $17 \mathrm{~K}^{6.7}$. Ferromagnetic behavior has also been observed in micro crystalline carbon 8 and micro graphite structures 9 . Theoretical efforts in understanding magnetism in C-based systems are being made. A ferromagnetic phase of mixed $s p^{2}$ and $s p^{3}$ pure carbon has been predicted theoretically 10 . Understanding the basic mechanism behind magnetic behavior of carbon-based materials and engineering novel ferromagnetic carbon structures are of prime importance.

Recently, Esquinazi et al 11 reported ferromagnetic (or ferrimagnetic) ordering of highly oriented pyrolitic graphite $(\mathrm{HOPG})$ with $\mathrm{T}_{c}$ above $300 \mathrm{~K}$ when irradiated with $2.25 \mathrm{MeV}$ protons. The possibility of occurrence of ferromagnetic, antiferromagentic and superconducting instabilities due to topological disorder in graphine sheets has also been predicted theoretically $\underline{12}$. It has been suggested that topological defects can be used to explain the observed ferromagnetism in $\mathrm{Rh}-\mathrm{C}_{60}$. According to a recent theoretical investigation by Vozmediano et al $\stackrel{13}{\text {, }}$, proton irradiation can produce large local defects which give rise to the appearance of local moments whose interaction can induce ferromagnetism in a large portion of the graphite sample.

The discovery of ferromagnetism in rhombohedral $\mathrm{C}_{60}$ polymers has opened up the possibility of a whole new family of magnetic fullerenes and fullerides. Here we present the results of magnetization measurements on pristine and $2 \mathrm{MeV}$ proton irradiated $\mathrm{C}_{60}$ films deposited on hydrogen passivated $\mathrm{Si}(111)$ surfaces. We find that magnetism is induced in proton irradiated $\mathrm{C}_{60}$ films.
Transmission electron microscopy (TEM), micro Raman, nuclear resonant scattering (NRS) and proton induced Xray emission (PIXE) measurements were used to characterize the samples. Magnetic measurements were carried out using a superconducting quantum interference device (SQUID) magnetometer.

The hydrogen passivation of $\mathrm{Si}(111)$ surfaces involves the following: degreasing of the Si substrate, removal of native oxide, and then growth of a thin uniform oxide, etching the oxide produced and passivating the surface with $\mathrm{H}$, using $40 \% \mathrm{NH}_{4} \mathrm{~F}$ solution. The detailed procedure is given in ref 14 . The passivated substrates $[\mathrm{H}-$ $\mathrm{Si}(111)]$ were loaded into a high vacuum $\left(4 \times 10^{-6}\right.$ mbar $)$ chamber and deposition of the fullerene film was carried out by evaporating $99.9 \%$ pure $\mathrm{C}_{60}$ [MER Inc., USA] from a tantalum boat.

Nuclear resonant scattering (NRS) measurements and ion irradiation were carried out using the $3 \mathrm{MV}$ 9SDH2 tandem Pelletron accelerator facility in our laboratory. We usually use Rutherford backscattering spectrometry (RBS) experiments to determine the film thickness. However for $\mathrm{C}$ the Rutherford scattering cross section is rather small. To enhance the scattering cross section, we choose appropriate energy of the incident ions and use a resonant scattering condition. A beam of $4.265 \mathrm{MeV}$ alpha particles was used for the NRS experiments to determine the film thickness. These measurements were carried out on a sample area of $8 \mathrm{~mm}^{2}$ and thickness is estimated to be $1.88 \mu \mathrm{m}$ from NRS. Then the $\mathrm{C}_{60}$ films were irradiated uniformly with a $2 \mathrm{MeV} \mathrm{H}^{+}$beam by rastering the ion beam on the sample. The ion fluence of the irradiated sample was $6 \times 10^{15} \mathrm{H}^{+} / \mathrm{cm}^{2}$. The beam current during irradiation was kept at $\sim 50 \mathrm{nA}$. Plan view TEM measurements were carried out using $200 \mathrm{keV}$ (JEOL 2010) HRTEM with point to point resolution of $0.19 \mathrm{~nm}$ and lattice resolution of $0.14 \mathrm{~nm}$. Raman spectra were recorded at room temperature using a Joven Yvon Raman spectrometer with Ar laser $\left(514 \mathrm{~cm}^{-1}, 25 \mathrm{~mW}\right.$ $\mathrm{cm}^{-2}$ ). Magnetization was measured using the reciprocating sample option (RSO) in a SQUID magnetometer (MPMS XL, Quantum Design) in the temperature range 
of $2-300 \mathrm{~K}$ in applied fields up to 7 Tesla.

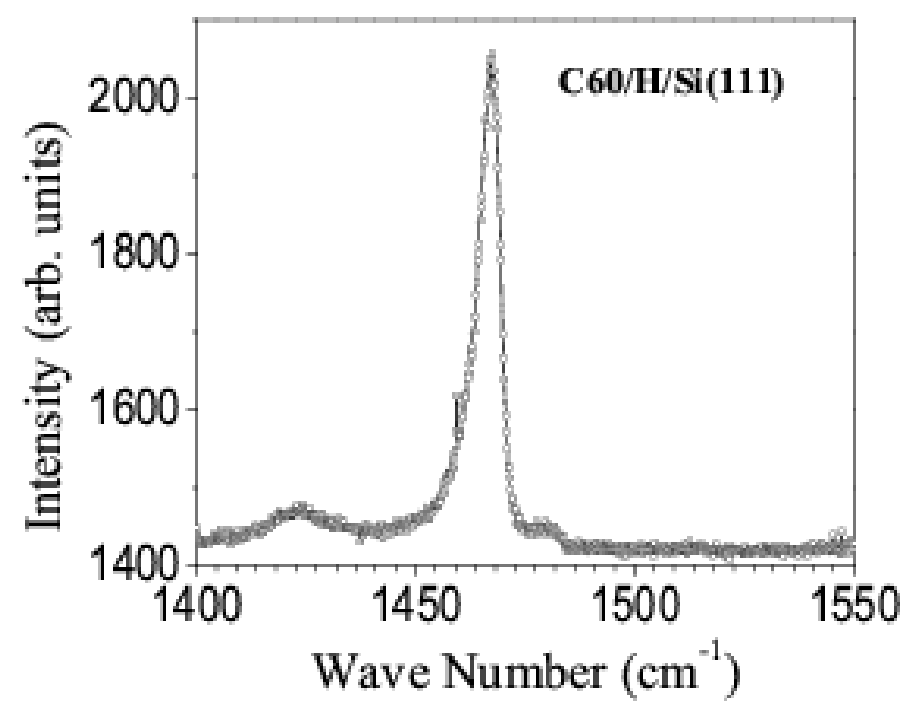

FIG. 1: A Raman spectrum of $\mathrm{C}_{60} / \mathrm{H}-\mathrm{Si}(111)$ film taken at room temperature.

A Raman spectrum from an as-deposited $\mathrm{C}_{60}$ film [Fig. 1] shows a strong peak at $1467 \mathrm{~cm}^{-1}$ which corresponds to the characteristic $\mathrm{A}_{g}$ mode of $\mathrm{C}_{60}{ }^{15}$. High resolution TEM (HRTEM) lattice images of as-deposited and irradiated samples with corresponding diffraction patterns are shown in Fig. 2. The transmission electron diffraction(TED) and HRTEM images confirm the crystalline nature of the $\mathrm{C}_{60}$ film in the fcc structure. Twin structures are seen in Fig. 2(a). Presence of defect structures in the irradiated film is evident from Fig 2(c). The (111) planar spacing of an fcc fullerene film is seen in Fig 2 (a) and $(\mathrm{c})$.

Results of magnetization vs. field $(\mathrm{M}-\mathrm{H})$ measurements at $5 \mathrm{~K}$ for the film irradiated at a fluence of $6 \times$ $10^{15} \mathrm{H}^{+} / \mathrm{cm}^{2}$ show a marked increase of magnetization and a tendency towards saturation [Fig. 3]. A weak remanent magnetization of the order of a few tens of $\mu \mathrm{emu}$ is observed (data not shown). At $300 \mathrm{~K}$, both the asdeposited and the irradiated sample show diamagnetic behavior. Magnetization data in the temperature range of $2-300 \mathrm{~K}$, in $1 \mathrm{~T}$ applied field, for the irradiated film shows much stronger temperature dependence when compared with that of the pristine film [Fig. 4].

The magnetic moment observed for the irradiated sample in an applied field of $7 \mathrm{~T}$ is about $5 \times 10^{-3} \mathrm{emu}$ with a tendency towards saturation. Magnetization curve of the irradiated film in high fields at $5 \mathrm{~K}$ compared with that of the as-deposited film and the empty substrate (the latter not shown here) gives clear evidence for the irradiation-induced magnetism in $\mathrm{C}_{60}$ films. The total amount of magnetic impurities ( $\mathrm{Fe}, \mathrm{Cr}, \mathrm{Ni}$ ) was determined by post-irradiation proton induced $\mathrm{X}$-ray emission
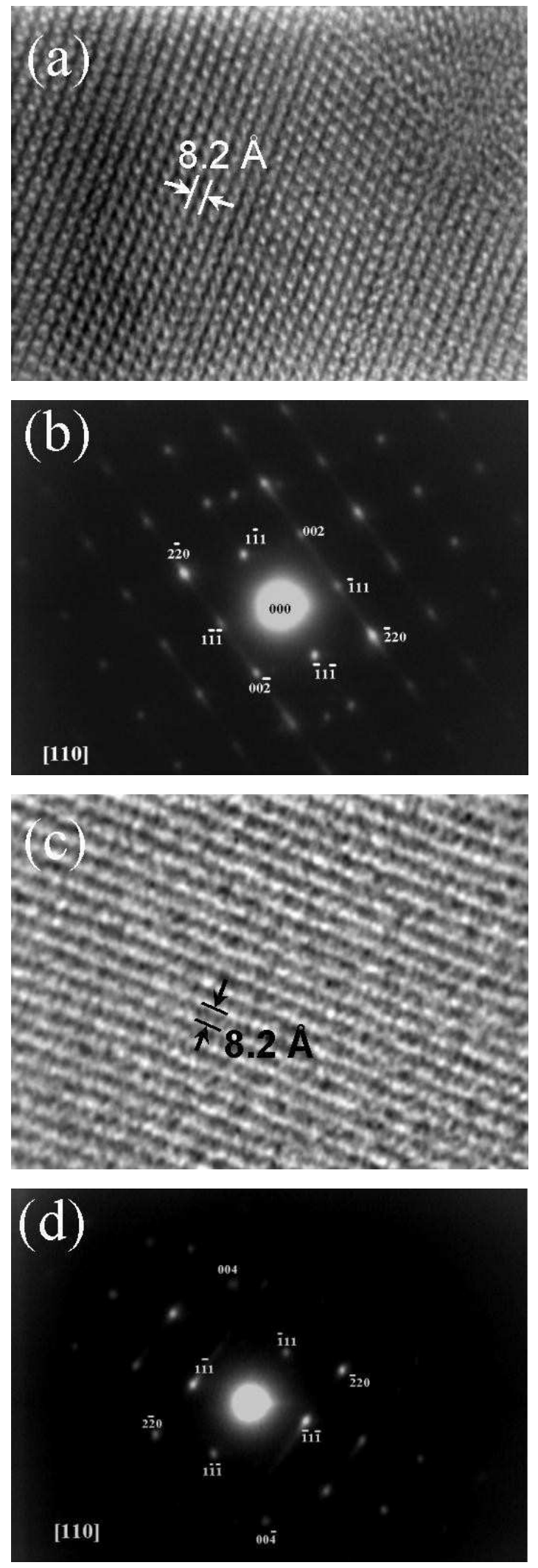

FIG. 2: (a) A high resolution TEM (HRTEM) lattice image from an as-deposited $\mathrm{C}_{60}$ film and (b) the corresponding selected area transmission electron diffraction (TED) pattern; (c) A HRTEM image from an irradiated $\mathrm{C}_{60}$ film and (d) the corresponding TED pattern. The (111) planar spacing of fcc fullerene is marked in (a) and (c). 


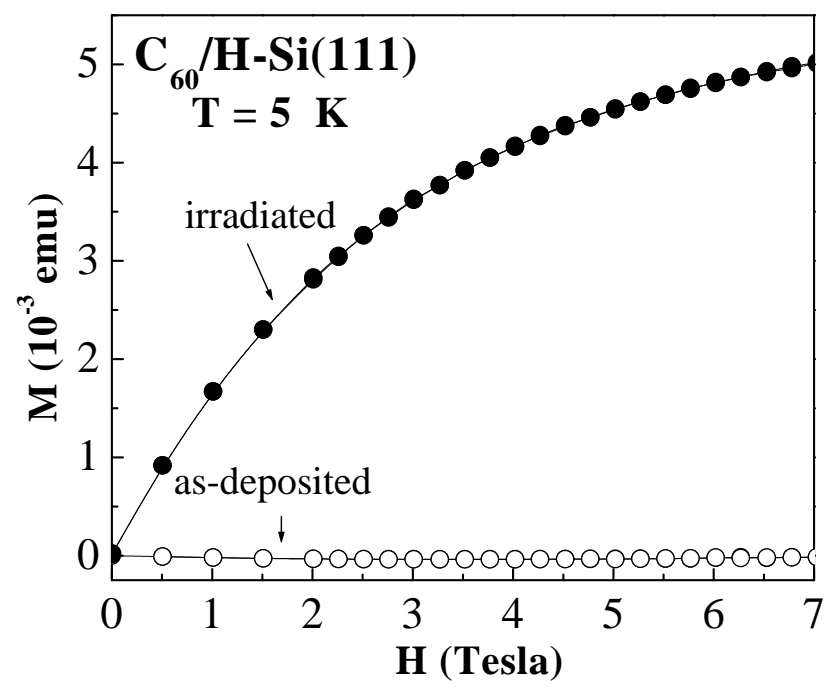

FIG. 3: $\mathrm{M}$ vs. $\mathrm{H}$ at $5 \mathrm{~K}$ for an as-deposited and an irradiated $\mathrm{C}_{60}$ film after subtracting the substrate [H-Si(111)] contribution (substrate data not shown here).

(PIXE) experiments and estimated to be $\sim 50 \mathrm{ppm}$ and the maximum magnetic moment contribution due to all these impurities in our film will be less than $5 \times 10^{-7}$ emu. Thus the contribution to observed magnetization due to these impurities is negligible. Saturation magnetization has been observed in pressure polymerised $\mathrm{C}_{60}$ films in applied fields of less than $2 \mathrm{~T}^{\underline{4}}$ whereas in the present svstem complete saturation is not attained even

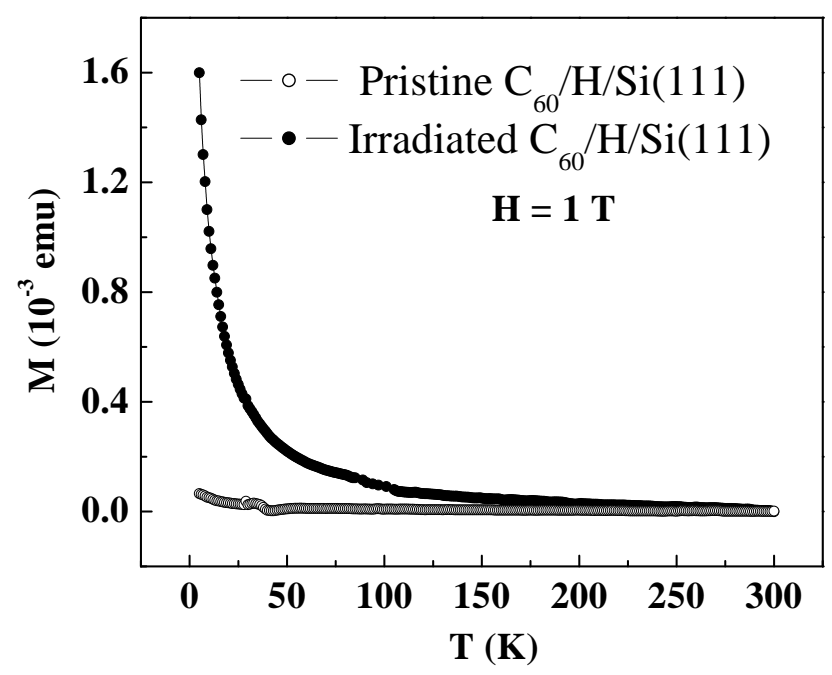

FIG. 4: $\mathrm{M}$ vs. $\mathrm{T}$ for an as-deposited and an irradiated $\mathrm{C}_{60}$ film in an applied field of $1 \mathrm{~T}$ (substrate [H-Si(111)] contribution subtracted).

The range of $2 \mathrm{MeV}$ protons, calculated by using SRIM $^{16}$ simulation programme for an amorphous carbon target having the density of $\mathrm{C}_{60}$, is found to be $\sim 50 \mu \mathrm{m}$.
Since our $\mathrm{C}_{60}$ film is only $\sim 1.9 \mu \mathrm{m}$ thick, the protons pass through the film and get buried deep into the $\mathrm{Si}$ substrate. The total energy loss of the proton beam in the present $1.9 \mu \mathrm{m}$ thick $\mathrm{C}_{60}$ film is $\sim 45 \mathrm{keV}$. The energy loss of protons at the top and the bottom of the $\mathrm{C}_{60}$ film are $24.4 \mathrm{eV} / \mathrm{nm}$ and $24.8 \mathrm{eV} / \mathrm{nm}$ respectively. As the proton energy loss is uniform over the whole depth of the film it is reasonable to assume that the irradiation damage is uniformly produced over the whole depth of the film. We have used the total thickness of the film in order to determine the magnetization value in $\mathrm{emu} / \mathrm{g}$.

The magnetization curve of $\mathrm{Rh}^{-} \mathrm{C}_{60} \frac{4}{4}$ shows a tendency to saturate even at an applied field of $2 \mathrm{~T}$, but for the present irradiated sample a tendency towards saturation can be seen at high fields. The magnetization at $7 \mathrm{~T}$ is about $200 \mathrm{emu} / \mathrm{g}$. (Similar large magnetization value (400 emu/g) had been reported for proton-irradiated HOPG samples ${ }^{17}$ ). In the proton-irradiated $\mathrm{C}_{60}$ films, although defects are created, the observation of ordered periodic lattice fringes in the irradiated sample and corresponding TED pattern indicate that irradiation did not cause disintegration of $\mathrm{C}_{60}$ cage leading to amorphization.

Regarding the mechanism for the formation of magnetic state in all-carbon systems, among others, the defect mediated mechanism appears to be the most general one. The defect-mediated mechanism has been addressed in a number of publications 4.5.12.13 Although the details may be different for different carbon systems (graphite, polymeric fullerene, nanotubes etc.), the common feature is the presence of undercordinated atoms, such as vacancies 18 , and atoms in the edges of graphitic nanofragments $19,20.21$. Ion irradiation of any materials generates vacancies. The enhancement in magnetization observed in $\mathrm{H}^{+}$-irradiated $\mathrm{C}_{60}$ samples may be due to defect moments from vacancies and/or deformation and partial destruction of fullerene cage. The HRTEM image in Fig. 2 (c) points to this possibility. There are reports 19.20 .21 that nanographitic fragments can trigger ferromagnetism. Formation of graphitic nanofragments in ion-irradiation of fullerene cannot be ruled out. Further studies like estimating the presence of nanographitic fragments and carbon vacancies in such systems will provide deeper insight into the understanding of origin of magnetism in the ion-irradiated $\mathrm{C}_{60}$ films.

According to a recent density functional study 18 of magnetism in proton irradiated graphite $\frac{11}{1}$, it is shown that $\mathrm{H}$-vacancy complex plays a dominant role for the observed magnetic signal. For a dose of $10 \mu \mathrm{C}$ the predicted signal is $0.8 \mu \mathrm{emu}$ which is in agreement with the experimental signal11. The implanted proton dose in our sample is $77 \mu \mathrm{C}\left(6 \times 10^{15}\right.$ ions $\left.\mathrm{cm}^{-2}\right)$ and all the protons are buried into silicon. So far we have not come across any report showing magnetic ordering in proton irradiated silicon. Even if we assume the same kind of magnetism due to $\mathrm{H}$-vacancy complex in proton-irradiated silicon as in proton-irradiated graphite, the expected magnetic signal would be three orders of magnitude smaller than our ob- 
served result. Considering the above fact we can safely ignore the contribution of implanted protons in the Si substrate to the observed magnetism, which is predominantly due to atomic displacements caused by energetic protons while passing through the film.

In conclusion, we have observed ferromagnetic-like behavior in $2 \mathrm{MeV}$ proton-irradiated $\mathrm{C}_{60}$ films. Magnetism in this irradiated films arises due to atomic displacements caused by the energetic protons as they pass through the film. Possible sources of magnetization are isolated vacancies, vacancy clusters or formation of nanographitic fragments. Further investigations are necessary to estimate their relative contribution.

We thank Prof. S. N. Behera for his important suggestions and a critical reading of the manuscript. Also we thank S. Rath and Prof. S. N. Sahu for Raman measurements and T. R. Rautray for PIXE data acquisition.
* Electronic address: bhupen@iopb.res.in

${ }^{1}$ For a recent review see, P. Esquinazi and R. Hohne arxive cond-mat/0409455 $/ 11 / \mathrm{sept} 2004 ; \mathrm{A}$. V. Rode et al. Phys. Rev. B 70, 54407 (2004).

2 J. Vcciana, $\pi$-Electron Magnetism: From Molecules to Magnetic Materials (Springer Verlag, Berlin 2001).

${ }^{3}$ V. I. Srdanov, G. D. Stucky, E. Lippmaa G. Engelhardt, Phys. Rev. Lett 80, 2449 (1998).

4 T. L. Makorova, B. Sundqvist, R. Hohne, P. Esquinazi, Y. Kopelevich, P. Scharff, V. A. Davydov, L. S. Kashevarova and A. V. Rakhmania, Nature 413, 716 (2001), and the references therein.

${ }^{5}$ R. A.Wood, M. H. Lewis, M. R. Lees, S. M. Bennington, M. G. Cain and N. Kitamura, J. Phys. Conds. Matter. 14, L385 (2002).

6 B. Narymbetov, A. Omerzu, V. V. Kabanov, M. Tokumoto, H. Kobayashi, D. Mihallovic, Nature 407, 883 (2000).

7 P. M. Allemand, K. C. Khemani, A. Koch, F. Wudl, K. Holczer, S. Donovan, G. Gruner, J. D. Thompson, Science 253, 301 (1991).

8 S. Mizogami, M. Mizutani, M. Fukuda, K. Kawabata, Synth. Met. 41-43, 3271 (1991).

9 C. Ishii, Y. Matsumura, K. Kaneko, J. Phys. Chem. 99, 5743 (1995); C. Ishii, N. Shindo, K. Kaneko, Chem. Phys. Lett. 242, 196 (1995).
10 A. A. Ovchinnikov, I. L. Shamovsky, J. Mol. Struct. (Theochem) 83, 133 (1991).

11 P. Esquinazi, D. Spemann, R. Hohne, A. Setzer, K. H. Han and T. Butz, Phys. Rev. Lett. 91, 227201 (2003).

12 J. Gonzalez, F. Guinea, M. A. H. Vozmediano, Phys. Rev. B 63, 134421 (2001).

13 M. A. H. Vozmediano, F. Guinea and M. P. Lopez-Sancho, arxive:cond-mat/040956v/22/sept2004.

14 S. Mathew, B. Satpati, B. Joseph and B. N. Dev, Appl. Surf. Sci. (in press).

15 J. Kastner, H. Kuzmany, L. Palmetshofer, P. Baur, G. Stingeder Nucl. Instr. and Meth. B 80/81, 1456 (1993).

16 SRIM 2003 - a version of the TRIM program: J. F. Ziegler, J. P. Biersack and U. Littmark The Stopping and Range of Ions in Matter (Perganon Press, New York, 1995).

17 K. H. Han, P. Esquinazi, J. Appl. Phys. 96, 1581 (2004).

18 P. O. Lehtinen, A. S. Foster, Y. Ma, A. V. Krasheninnikov, R. M. Nieminen, Phys. Rev. Lett. 93, 187202 (2004).

19 P. Esquinazi, A. Setzer, R. Hohne, C. Semmelhak, Y. Kopelevich, D. Spemann, T. Butz, B. Kohlstrunk, and M. Losche, Phys. Rev. B 66, 24429 (2002).

${ }^{20}$ K. Nakada, M. Fujita, G. Dresselhaus and M. S. Dresselhaus, Phys. Rev. B 54, 17954 (1996).

${ }^{21}$ H. Lee, N. Park, Y. W. Son, S. Han, J. Yu, Chem. Phys. Lett. 398, 207 (2004). 